\title{
A Cross-Docking Framework for Supply Chain Logistics in Production Scheduling
}

\author{
Hongnian $\mathrm{Yu}^{1}$ and Gyan Bahadur Thapa ${ }^{2}$ \\ ${ }^{1}$ Faculty of Science and Technology, Bournemouth University, UK \\ ${ }^{2}$ Institute of Engineering, Tribhuvan University, Kathmandu, Nepal
}

\begin{abstract}
Synchronization of all partners along a supply chain logistics system is one of the challenges in most of the business operations. In this paper, we present a brief literature review of cross-docking approaches for supply chain logistics problem. On describing the problem, we formulate the supply chain logistics problem as a truck sequencing problem to minimize the discrepancies of operation times of inbound and outbound trucks. The problem is decomposed in two sub-problems as inbound and outbound parts as a solution strategy.
\end{abstract}

Key words: Decomposition, truck sequencing, supply chain, cross-docking logistics, makespan

\section{INTRODUCTION}

Today's business challenge lies in supplying the products of excellent quality, lowest cost and in-time delivery to achieve customer satisfaction, and in staying ahead of competitors in market activities. Generating the optimal production schedule for an assembly line to balance the overall supply chain under a variety of practical constraints is a difficult task. Many companies are attempting to make their production system more flexible or adaptable with change to respond the varying customer demands. One of the most significant concepts in production industries in past decades has been just-intime (JIT) production system, which is a philosophy as much as a technique based upon the idea that no activity should take place until there is a need for it. JIT system is a pull concept where demand pulls goods towards the market. A supply chain is the network of organizations, people, activities, information and resources involved in the physical flow of products from initial suppliers to final customers. Several researches have been carried out in production and logistic networks independently. However, many decisions in production and logistics need to be addressed simultaneously while minimizing the costs associated with production, warehousing and transportation. The mutual coordination among independent firms (viz., raw-material suppliers, manufacturers, distributors and retailers) is the crux to attain the flexibility required to enable them in the progressive improvement of logistics processes. Poor coordination among the chain members creates poor performance, such as higher inventory and transportation costs, longer delivery times, higher levels of loss or damage and lower customer service (Lee et al. 1997). The proper collaboration among all components of production and distribution networks can minimize the cost and maximize the benefits for all. To realize the best quality production and timely distribution in dynamic environment with proper coordination, it is essential to create a cross-docking environment throughout the supply chain system that is capable to address the diversified demands.

\section{Cross-Docking Logistics}

The cross-docking (CD) logistics is the movement of products directly from receiving dock to shipping dock with minimum dwell time in between. It is one of the new logistics techniques used in retail and trucking industries to move materials from inbound locations to outbound locations as quickly as possible. By arranging immediate $\mathrm{CD}$ of incoming products, retailers are able to minimize in-transit time for their incoming products. Since the introduction of the term supply chain management in 1982 (Oliver \& Webber 1992), it has received ever-growing interest both in the literature and in industrial practice. The JIT production logistics is a specific part of the supply chain that deals with the control of materials and information flows within the supply chain of manufacturing companies with the mission to get the right materials to the right place at the right time in perfect quality at the lowest possible costs (Ghiani et al. 2004, Thapa \& Dhamala 2009). The JIT logistics is performed to optimize some sort of given performance measures (e.g., minimizing total operating costs) and to satisfy a given set of constraints (e.g., budget constraints). The JIT production and delivery systems uses a set of integrated activities to achieve flexibility in manufacturing with minimum shortages and inventories. The supply chain synchronization is 
shown in Fig.1 in terms of inbound and outbound logistics.

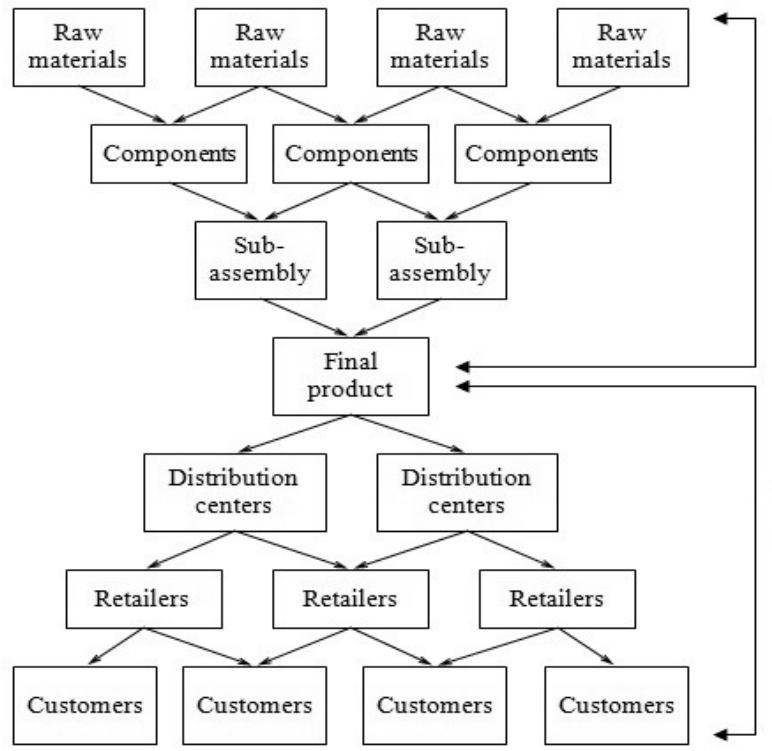

Fig. 1. Supply chain network synchronization

Many companies are trying to develop efficient methods to increase customer satisfaction and to reduce costs. The $\mathrm{CD}$ is considered as one of the best methods to reduce inventory and improve responsiveness to various customer demands. The products are received in a distribution center occasionally merged with other products going to the same destination, then shipped at the earliest opportunity without putting away, storing and picking as shown in Fig. 2. The benefits of CD include low inventory, low handling costs and space, low transportation costs. Buffa (1986) showed that logistics cost could be reduced by integrating the inbound and outbound vehicles in the distribution system. Uday and Viswanathan (2000) provided a framework for understanding and designing CD systems discussing the techniques to improve efficiency of the logistics and distribution operations. The role of information logistics in supply chain process is studied in Thapa et al. (2010). Li et al. (2004) considered material handling inside the terminal for a given truck schedule. Once a set of inbound and outbound trucks is docked, jobs consisting of products to be handled should be assigned to resources in such a way that efficient unloading, sorting and loading operations render possible. So far, McWilliams et al. (2005) and Yu \& Egbelu (2006) have only dealt the truck scheduling problem. The former groups covered a specific truck scheduling problem at a parcel hub which is solved by a simulation-based optimization approach, and the latter ones treated a special kind of $\mathrm{CD}$ terminal with a conveyor belt system, where a single inbound door serves a single outbound door.

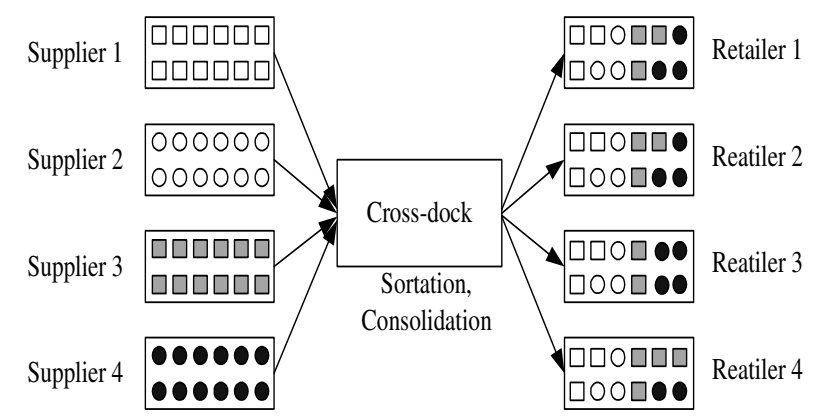

Fig. 2. Freight consolidation at the transfer terminal

Boysen et al. (2010) dealt a stylized "one inbound door serves one outbound door" setting to generate fundamental insights to the underlying real-world problem structure. Exact handling times for inbound trailers depend on the exact packing of goods and the sequence in which they can be obtained, whereas those for outbound trailers have to account for load stability and the sequence in which customers are served. A time-slot comprises the time required for completely unloading an inbound truck and completely loading an outbound truck respectively. Handling times in between dock doors are considered by a delay (measured in number of slots) covering the time span until incoming products are available at an outbound door.

\section{Problem Description}

We use the following notations to describe the $\mathrm{CD}$ logistics as truck sequencing problem (TRSP): let $I$ and $O$ be the sets of inbound and outbound trucks respectively at the single receiving door and the single shipping door of the $\mathrm{CD}$ terminal. Each inbound truck is loaded with units of different products $p \in P$. Let $a_{\alpha p}$ and $b_{\beta p}$ be the number of units of product type $p$ arriving in inbound truck $\alpha$ and to be loaded onto outbound truck $\beta$ respectively. All product units are completely unloaded within a service slot $t$ to which the respective inbound truck is assigned, so that all handling operations (docking, unloading and undocking) required to process the truck, are executed within this time span, e.g., an hour or two. All inbound trucks are assumed to be available for processing at the beginning of planning horizon, so that a static problem with identical arrival dates of inbound trucks is considered. The assumption of equally long service times can be seen as a reasonable approximation of reality, whenever vehicle capacities and the number of products per vehicle do not strongly differ. As trailers are typically of a standardized size and 
CD aims to move only full truck loads, this premise is fulfilled whenever all processed products are of comparable size (e.g., mail distribution centers) or all truck loads resemble a representative average truck load (e.g., rotational deliveries of special promotional offers to all stores of a chain).

The delivered products have to undergo several subsequent operations before they are available for loading onto the outbound trucks at the shipping door. These operations include recording of any product unit in the information system, examining the product correctness and quality, sorting, rearranging and packing to recombine products from different inbound trucks to form the load of a certain outbound truck. Finally, the products have to be transported to the shipping door, where they wait in an intermediate buffer of sufficient size. This task from recording to transporting is assumed to last a fixed movement time $m$ irrespective of the truck load. Then all products arriving in a slot $t$ are available for loading at the shipping dock not before slot $t+m$. If the movement starts not before the complete inbound truck has been unloaded, the units are first available at slot $t+m+1$. For constant unloading times, it is assumed that the movement time $m$ is independent of the inbound truck and the loaded products.

At the shipping door, the set of outbound trucks is to be loaded, each $\beta \in O$ with a predetermined number of units $b_{\beta p}$ of the different products $p \in p$. It is assumed that all handling operations per truck are completed within a single slot. An outbound truck can be assigned to a slot $t$ not before enough stock has accumulated in the intermediate buffer to serve all demanded product units of the truck. As only temporary stock is allowed within a cross dock, it is assumed that temporary stock is empty before the first inbound truck arrives and is emptied out again after the last outbound truck was served. This means $\sum_{\alpha \in I} a_{\alpha p}=\sum_{\beta \in O} b_{\beta p}, \forall p \in P$. The simplifying assumptions applied to our base model are summarized as follows (Boysen et al. 2010):

- Inbound trucks are processed at a single receiving door of the terminal that serves a single shipping door for outbound trucks; doors are distinct. And the processing trucks (loading or unloading) takes the same amount of time.

- No predefined restrictions on truck assignments to slots exist, e.g., release or due dates.

- The input data is known in advance with certainty (static deterministic).

- The sum of units delivered by inbound trucks equals the sum of units consumed by outbound trucks for any product $p$. And intermediate buffer for intermediate stock is not limited in size.

\section{Problem Formulation}

Let $I=$ set of inbound trucks indexed by $\alpha, O=$ set of outbound trucks indexed by $\beta, P=$ set of products indexed by $p, T=$ total number of time slots indexed by $t, a_{\alpha p}=$ quantity of $p$ arriving in truck $\alpha, b_{\beta p}=$ quantity of $p$ to be loaded in truck $\beta$; and let

$$
\begin{aligned}
x_{\alpha t}= & 1, \text { if inbound truck } \alpha \text { is assigned to slot } t \\
& 0, \text { if otherwise } \\
y_{\beta t}= & 1, \text { if outboundtruck } \beta \text { is assignedto slot } t \\
& 0, \text { if otherwise }
\end{aligned}
$$

As a result of above assumptions, the inbound and outbound schedule can be readily derived by the sequence of inbound and outbound trucks. The problem is to sequence the trucks such that the operation time (called makespan) is minimized which comprises the time span starting from the first slot to which an inbound truck is assigned and lasts until the final slot in which an outbound truck is processed. The TRSP is formulated as follows:

Minimize $M=\sum_{t=1}^{T} t x_{\alpha t}-t y_{\beta t} \mid, \forall \alpha \in I$ and $\beta \in O$

subject to

$$
\begin{aligned}
& \sum_{t=1}^{T} x_{\alpha t}=1, \quad \forall \alpha \in I \\
& \sum_{\alpha \in I} x_{\alpha t} \leq 1, \quad \forall t=1,2, \ldots, T \\
& \sum_{t=1}^{T} y_{\beta t}=1, \quad \forall \beta \in O \\
& \sum_{\beta \in O} y_{\beta t} \leq 1, \quad \forall t=1,2, \ldots, T \\
& \sum_{\tau=1}^{t} \sum_{\alpha \in I} x_{\alpha \tau} \cdot a_{\alpha p} \geq \sum_{\tau=1}^{t} \sum_{\beta \in O} y_{\beta \tau} \cdot b_{\beta p}, \\
& \forall t=1,2, \ldots, T ; p \in P \\
& x_{\alpha t} \in\{0,1\}, \forall \alpha \in I ; t=1,2, \ldots, T \\
& y_{\beta t} \in\{0,1\}, \quad \forall \beta \in O ; t=1,2, \ldots, T
\end{aligned}
$$

The objective (1) minimizes the sum of the absolute difference of operation times of outbound trucks $\beta$ and inbound trucks $\alpha$. The constraint (2) ensures that each inbound truck is processed in exactly one slot, whereas constraints (3) enforces that in each slot at most one inbound truck can be assigned. In analogy, these two conditions also hold for outbound trucks by constraints (4) and (5). Constraints (6) ensure that an outbound 
truck can only be assigned to a slot $t$ whenever all required products are available (delivered by preceding inbound trucks yet not consumed by preceding outbound trucks) to satisfy the demand for product units of each type $p$. Therefore, the available stock accumulated by all inbound trucks assigned to slots $\tau=1, \ldots, t$ has to exceed the total demand for product units of outbound trucks scheduled up to the actual slot $t$ (note that this will actually be slot $t+m$ or even $t+m+1$ when realizing the schedule). The constraints (7) and (8) represent the binary variables for inbound and outbound trucks respectively.

As the makespan is to be minimized, the number of service slots actually required is unknown prior to the solution of the model. That is, the number of slots $T$ in the model is to be initialized with some upper bound $B$ on the makespan: $T=B$. One simple upper bound is given by $B=|I|+|O|-1$. This bound is based on the consideration that in the worst case the first outbound truck scheduled requires a product loaded on the last inbound truck scheduled. Consequently, all outbound trucks have to wait until all inbound trucks are unloaded. To tighten the model formulation, e.g., when using a generic MIP-solver, the following property of optimal inbound schedules can be utilized (Boysen et al. 2010), by which number of variables and constraints can be reduced:

Left-shift property: It is sufficient to reduce the set of time slots considered for an assignment of inbound trucks to the first $|I|$ slots. This is obviously correct, because if there exists an optimal solution, where inbound trucks are not assigned to slots $t=1, \ldots,|I|$ in direct succession, then trucks can be brought forward (without altering the sequence) and the objective value remains the same. Thus, there is always at least one optimal solution where inbound trucks are assigned to the first $|I|$ slots. As alterations are truly straight forward we abstain from explicitly recording them.

\section{Lower Bounds}

The first simple lower bound $B_{1}$ reverses the logic of our upper bound. In the best case, each outbound truck has a direct counterpart among the inbound trucks, so that inbound and outbound trucks can be scheduled successively without any delay:

$$
B_{1}=\max \{|I| ;|O|\}
$$

For the computation of another lower bound $B_{2}$, the overall problem is decomposed in $|P|$ sub-problems by cutting off the truck coherency of products. For each product the minimum makespan is deduced by separately scheduling inbound and outbound trucks. Thus, it is relaxed that for each product the same truck sequence has to be maintained. The optimal solution for each sub-problem can be determined by considering the following simple rules, which share some similarities with those of the famous Johnson algorithm for the twomachine flow shop scheduling problem (Johnson, 1954):

- Sort the set $I$ with respect to descending loads $a_{\alpha p}$ of product $p$ actually considered. This leads to a sequence vector $\pi^{p}$ with elements $\pi_{\alpha}^{p},(\alpha=1, \ldots$, $|I|$ I). The schedule for this sequence is readily available because of the left-shift property: Inbound trucks are scheduled according to sequencing vector $\pi^{p}$ in direct succession starting with slot $t=1$.

- Sort the set $O$ of outbound trucks with respect to ascending loads $b_{\alpha p}$ of the actual product $p$. This sequence is stored in the vector $\mu^{p}$ with elements $\mu_{\beta}^{p},(\beta=1, \ldots,|O|)$. The resulting slots $s_{p \beta}$ can be computed by assigning, in each case, the first feasible slot number $t$ according to:

$$
s_{\beta p}=\min \left\{t=s_{\beta-1 p}+1, \cdots, T: \sum_{\tau=1}^{\min \{I \mid, t\}} a_{\pi \tau}^{p} p \geq \sum_{\tau=1}^{\beta} b_{\mu \tau}^{p} p,\right\} \ldots \ldots . .(10)
$$

To initialize the recursive formulae (10), a slot $\mu_{\beta}^{p}$ has to be initialized with slot number $\beta$. The lower bound $B_{2}$ then simply amounts to the maximum makespan over all products

$$
B_{2}=\max _{p \in P}\left\{s_{|O| p}\right\}
$$

The lower bound $B_{2}$ has a runtime complexity of $\mathrm{O}(n$ $\log n$ ), where $n=\max \{|I| ;|O|\}$, due to the sorting operations, which is considerably higher than that of $B_{1}$. However, it can be shown that both bounds in any case lead to the same result (Boysen et al. 2010).

\section{Decomposition: A Solution Strategy for TRSP}

The TRSP problem is decomposed into two subproblems, namely inbound and outbound TRSPs, written as IBD-TRSP and OBD-TRSP respectively. It is divided into two sub-problems by fixing a particular inbound (outbound) sequence and then finding the optimal outbound (inbound) sequence respectively. In the following, we formalize the considered subproblems and identify a strong structural relationship that can be exploited in the solution procedure.

In the first problem let us assume that there is a fixed sequence $\pi$ of inbound trucks given, so that the inbound schedule can immediately be deduced by assigning the trucks in the respective order to the first $|I|$ slots (see left-shift property). Thus, the number $A_{t p}$ of product 
units available for outbound trucks is known in advance for each slot $t: A_{t p}=\sum_{\tau=1}^{\min \{|I| ; t\}} a_{\pi_{\tau}} p, \quad \forall p \in P ; t=$ $1,2, \ldots, T$. With the available stock (cumulated input) on hand, the problem OBD-TRSP reduces to objective function (1) subject to constraints (4), (5), (8) and (12):

$$
\begin{array}{r}
A_{t p} \geq \sum_{\tau=1}^{t} \sum_{\beta \in O} y_{\beta \tau} . b_{\beta p} \quad \ldots \ldots \ldots \ldots \\
\forall t=1,2, \ldots, T ; p \in P
\end{array}
$$

Note that this sub-problem is NP-hard in the strong sense. Boysen et al. (2010) proposed an exact dynamic programming approach and a priority rule based heuristic start procedure to solve OBD-TRSP. Conversely, the sequence $\mu$ of outbound trucks can be fixed starting from period $T$ to earlier ones and be determined the optimal sequence of inbound trucks, respectively. However, this requires additional modifications to the mathematical model. Note that in the original model, objective function (1) was defined in such a way, that it minimizes the index number of the service slot to which the last outbound truck in the sequence is assigned. As the outbound sequence is now not variable anymore, the objective function needs some adjustment. Makespan minimization can be readily expressed in terms of the inbound sequence by maximizing the first service slot to which any inbound truck is assigned. Let this slot in an optimal solution be denoted by $t^{*}$, then the first $t^{*}-1$ slots, to which no inbound trucks are assigned, can be discarded and the minimum makespan is equal to $T-t^{*}+1$. It can be easily verified that both objectives lead to the same optimal inbound and outbound sequences.

Instead of defining IBD-TRSP as a maximization problem, we take a slightly different approach which reveals an interesting relationship between IBD- and OBD-TRSP. Recall that in the original model, index $t$ denotes the index number of a service slot in ascending order, so that a lower number indicates that the service slot is processed prior to a slot with a higher number, which is an intuitive representation of time. For IBDTRSP, we will change the point of reference and introduce a new time index $j=1, \ldots, T$, where $j=1$ refers to the service slot to which the last outbound truck is assigned and an increase in $j$ denotes a movement backwards in time until service slot $T$, which constitutes the earliest point in time to which any inbound or outbound truck could be assigned. Consequently, any original index $t$ corresponds to $j=T-t+1$. The IBDTRSP can now be formulated as follows (Boysen et al. 2010):

(IBD-TRSP):Minimize

$$
M_{I B D}=\max _{\alpha \in I ; j=1, \ldots, T}\left\{x_{\alpha j} \cdot j\right\}
$$

subject to

$$
\begin{aligned}
& \sum_{j=1}^{T} x_{\alpha j}=1, \quad \forall \alpha \in I \\
& \sum_{\alpha \in I} x_{\alpha j} \leq 1, \forall j=1,2, \ldots, T \ldots \ldots \ldots \ldots \ldots \\
& M_{p}-\left(\sum_{\tau=1}^{j} \sum_{\alpha \in I} x_{\alpha \tau} \cdot a_{\alpha p}\right) \geq M_{p}-B_{j p} \\
& \forall j=1, \ldots, T ; p \in P \\
& x_{\alpha j} \in\{0,1\}, \forall \alpha \in I ; j=1,2, \ldots, T
\end{aligned}
$$

The objective function (13) minimizes the makespan in terms of the inbound sequence by minimizing the number of service slots between the last outbound and first inbound truck assigned. The constraints (14), (15) and (16) are simply modified according to the new time index $j$. The constraint (16) ensures that inbound trucks deliver product units in the required quantities, where $B_{j p}=\sum_{\tau=1}^{\min \{|O| ; j\}} b_{\mu T-\tau+1 p}, \forall p \in P ; j=1,2, \ldots, T$ denotes the total number of units of product $p$ demanded by the last $j$ outbound trucks in the fixed sequence $\mu$ and $M_{p}=\sum_{\alpha \in I} a_{\alpha p}=\sum_{\beta \in O} b_{\beta p}$ is the total number of parts delivered or demanded. Note that the constraint (16) can be rewritten as follows:

$$
B_{j p} \geq \sum_{\tau=1}^{j} \sum_{\alpha \in I} x_{\alpha \tau} \cdot a_{\alpha p}, \forall j=1,2, \ldots, T ; p \in P \ldots \ldots
$$

A comparison of IBD-TRSP and OBD-TRSP now reveals that their mathematical structures are identical. As a consequence, any algorithm for OBD-TRSP can be used to solve IBD-TRSP and vice-versa. In fact, IBDTRSP can be seen as a reverted OBD-TRSP in the sense that the solution of an instance of IBD-TRSP with an algorithm designed for OBD-TRSP requires following steps:

- $\quad$ Revert the given outbound sequence $\mu$ and set it as modified inbound sequence $\pi$. Consider the set of inbound trucks $I$ to be scheduled as the modified set of outbound vehicles $O$

- Solve OBD-TRSP with the modified input data.

- The reverted optimal outbound sequence constitutes the optimal inbound sequence for the original IBDTRSP instance.

Boysen et al. (2010) introduced an exact dynamic programming approach and a heuristic starting procedure to solve the identified sub-problems of TRSP. The algorithmic descriptions are limited to OBD-TRSP, as they are directly transferable to IBD-TRSP as explained above. Note that the general TRSP is NP-hard in the strong sense (Boysen et al. 2010). The supply chain logistics problem is studied via radio frequency 
identification (RFID) to determine the direction of the transportation vehicles (Choosri et al. 2013) with some experimental demonstrations. The cross-docking supply chain logistics problem is studied together with the multi-level JIT production environment in terms of mathematical models in both the cases, proposing an if and only if condition in balancing the solution of either problem (Thapa et al. 2010).

\section{CONCLUSION}

We have presented a model of makespan minimization problems in terms of absolute difference of operation times of inbound and outbound trucks. Our model is slightly different with that of Boysen model, which considers the operation time of outbound truck. A supply chain logistics problem is the multi-level distribution problem. The mutual coordination between production line and distribution line plays an important role in the overall supply chain management of the company. Balancing the production levels depends on the balancing of distribution levels, which is one of the rich research areas in production logistics. The simultaneous study of multi-level JIT production and distribution will be the focus of our further study.

\section{REFERENCES}

Boysen, N., Fliedner, M. and Scholl, A. 2010. Scheduling inbound and outbound trucks at crossdocking terminals. OR Spectrum 32:135-161.

Buffa, F.P. 1986. Inbound logistics: Analysing inbound consolidation opportunities. International Journal of Physical Distribution and Materials Management 16:3-32.

Choosri, N., Yu, H. and Atkins, A. 2013. Practical aspects of using passive UHF RFID technology for vehicle tracking. Int. J. Agile Systems and Management 6(1):43-65.

Ghiani, G., Laporte, G. and Musmanno, R. 2004. Introduction to logistics systems planning and control. John Wiley and Sons Ltd.

Johnson, S.M. 1954. Optimal two - and three - Stage production schedules with setup times included. Naval Research Logistics 1:61-68.

Lee, H.L., Padmanabhan, V. and Whang, S. 1997. The bullwhip effect in supply chains. Sloan Management Review 38:93-102.

Li, Y., Lim, A. and Rodrigues, B. 2004. Cross-docking JIT scheduling with time windows. Journal of the Operations Research Society 55:1342-1351.
McWilliams, D.L., Stanfield, P.M. and Geiger, C.D. 2005. The parcel hub scheduling problem: A simulation-based solution approach. Computer Industrial Engineering 49:393-412.

Oliver, R.K. and Webber, M.D. 1992. Supply chain management: logistic catches up with strategy (reprint of 1982). In: Christopher, M. (Ed.), logistics-the strategic issues, London, 63-75.

Stadtler, H. 2002. Basics of supply chain management. In: Stadtler, H., Kilger, C. (Eds.), Supply Chain Management and Advanced Planning- Concepts, Models, Software and Case Studies, Berlin, 7-28.

Stadtler, H. 2005. Supply chain management and advanced planning-basics, overview and challenges. European Journal of Operational Research 163:575-588.

Thapa, G.B. 2012. Characterizations of just-in-time sequencing problem with apportionment and supply chain. $\mathrm{PhD}$ Thesis, Tribhuvan University, Kathmandu, Nepal.

Thapa, G.B. and Dhamala, T.N. 2009. Just-in-time sequencing in mixed-model production systems relating with fair representation in apportionment theory. The Nepal Mathematical Sciences Report 29:29-68.

Thapa, G.B., Dhamala, T.N. and Yu, H. 2010. Mathematical model of cross-docking operation in supply chain under multi-level just-in-time production environment. The Nepali Mathe matical Sciences Report 30(1 \& 2): 24-35.

Thapa, G.B., Dhamala, T.N. and Yu, H. 2010. Role of informed logistics in supply chain productIon process. International Workshop on Human Adaptic Mechatronics, Loughborough University, UK 2010.

Uday, M.A. and Viswanathan, S. 2000. Effective crossdocking for improving distribution efficiencies. International Journal of Logistics: Research and Applications 33: 291-302.

Yu, W. and Egbelu, P.J. 2008. Scheduling of inbound and outbound trucks in cross-docking systems with temporary storage. European Journal of Operations Research 184: 377-396. 